\title{
Variance estimation in the particle filter
}

\author{
Anthony Lee and Nick Whiteley \\ University of Warwick and University of Bristol
}

June 29, 2016

\begin{abstract}
This paper concerns numerical assessment of Monte Carlo error in particle filters. We show that by keeping track of certain key features of the genealogical structure arising from resampling operations, it is possible to estimate variances of a number of standard Monte Carlo approximations which particle filters deliver. All our estimators can be computed from a single run of a particle filter with no further simulation. We establish that as the number of particles grows, our estimators are weakly consistent for asymptotic variances of the Monte Carlo approximations and some of them are also non-asymptotically unbiased. The asymptotic variances can be decomposed into terms corresponding to each time step of the algorithm, and we show how to consistently estimate each of these terms. When the number of particles may vary over time, this allows approximation of the asymptotically optimal allocation of particle numbers.
\end{abstract}

\section{Introduction}

Particle filters, or sequential Monte Carlo methods, provide Monte Carlo approximations of integrals with respect to sequences of measures. In popular statistical inference applications, these measures arise naturally from conditional distributions in hidden Markov models, or are constructed artificially to bridge between target distributions in Bayesian analysis. The numbers of particles used to perform the approximation controls the tradeoff between computational complexity and accuracy. Theoretical properties of this relationship have been the subject of intensive research; the literature includes a number of central limit theorems [Del Moral and Guionnet, 1999, Chopin, 2004, Künsch, 2005, Douc and Moulines, 2008] and a variety of refined asymptotic [Douc et al., 2005, Del Moral et al., 2007] and non-asymptotic [Del Moral and Miclo, 2001, Cérou et al., 2011] results. These studies provide a wealth of insight into the mathematical behaviour of particle filter approximations and validate them theoretically, but considerably less is known about how, in practice, to extract information from a realization of a single particle filter in order to report numerical measures of Monte Carlo error. This is in notable contrast to other families of Monte Carlo techniques, especially Markov chain Monte Carlo, for which an extensive literature on variance estimation exists. Our main aim is to address this gap.

We introduce particle filters via a framework of Feynman-Kac models [Del Moral, 2004]. This approach allows us to identify the key generic ingredients defining particle filters and the measures they approximate, and emphasizes that our variance estimators can be used across application areas. Based on a single realization of a particle filter, we provide unbiased estimators of the variance and individual asymptotic variance terms for a class of unnormalized particle approximations. No estimators of these quantities based on a single run of a particle filter have previously appeared in the literature. Upon suitable rescaling, we establish that our estimators are weakly consistent for asymptotic variances associated with a larger class of particle approximations. One of these re-scaled estimators is closely related to that proposed by Chan and Lai [2013], which is the only other consistent asymptotic variance estimator based on a single realization of a particle filter in the literature. We also demonstrate how 
one can use the estimators to inform the choice of algorithm parameters in an attempt to improve performance.

\section{Particle filters}

\subsection{Notation and conventions}

For a generic measurable space $(\mathrm{E}, \mathcal{E})$, we denote by $\mathcal{L}(\mathcal{E})$ the set of $\mathbb{R}$-valued, $\mathcal{E}$-measurable and bounded functions on $\mathrm{E}$. For $\varphi \in \mathcal{L}(\mathcal{E}), \mu$ a measure and $K$ an integral kernel on $(\mathrm{E}, \mathcal{E})$, we write $\mu(\varphi)=$ $\int_{\mathrm{E}} \varphi(x) \mu(\mathrm{d} x), K(\varphi)(x)=\int_{\mathrm{E}} K\left(x, \mathrm{~d} x^{\prime}\right) \varphi\left(x^{\prime}\right)$ and $\mu K(A)=\int_{\mathrm{E}} \mu(\mathrm{d} x) K(x, A)$. Constant functions $x \in$ $E \mapsto c \in \mathbb{R}$ are denoted simply by $c$. For $\varphi \in \mathcal{L}(\mathcal{E}), \varphi^{\otimes 2}\left(x, x^{\prime}\right)=\varphi(x) \varphi\left(x^{\prime}\right)$. The Dirac measure located at $x$ is denoted $\delta_{x}$. For any sequence $\left(a_{n}\right)_{n \in \mathbb{Z}}$ and $p \leq q, a_{p: q}=\left(a_{p}, \ldots, a_{q}\right)$. For any $m \in \mathbb{N},[m]=\{1, \ldots, m\}$. For a vector of positive values $\left(a_{1}, \ldots, a_{m}\right)$, we denote by $\mathcal{C}\left(a_{1}, \ldots, a_{m}\right)$ the Categorical distribution over $\{1, \ldots, m\}$ with probabilities $\left(a_{1} / \sum_{i=1}^{m} a_{i}, \ldots, a_{m} / \sum_{i=1}^{m} a_{i}\right)$. When a random variable is indexed by a superscript $N$, a sequence of such random variables is implicitly defined by considering each value $N \in \mathbb{N}$, and limits will always be taken along this sequence.

\subsection{Discrete time Feynman-Kac models}

On a measurable space $(\mathrm{X}, \mathcal{X})$ with $n$ a non-negative integer, let $M_{0}$ be a probability measure, $M_{1}, \ldots, M_{n}$ a sequence of Markov kernels and $G_{0}, \ldots, G_{n}$ a sequence of $\mathbb{R}$-valued, strictly positive, upper-bounded functions. We assume throughout that $X$ does not consist of a single point. We define a sequence of measures by $\gamma_{0}=M_{0}$ and, recursively,

$$
\gamma_{p}(S)=\int_{\mathbf{X}} \gamma_{p-1}(\mathrm{~d} x) G_{p-1}(x) M_{p}(x, S), \quad p \in[n], \quad S \in \mathcal{X} .
$$

Since $\gamma_{p}(\mathbf{X}) \in(0, \infty)$ for each $p$, the following probability measures are well-defined:

$$
\eta_{p}(S)=\frac{\gamma_{p}(S)}{\gamma_{p}(\mathrm{X})}, \quad p \in\{0, \ldots, n\}, \quad S \in \mathcal{X} .
$$

The representation

$$
\gamma_{n}(\varphi)=E\left\{\varphi\left(X_{n}\right) \prod_{p=0}^{n-1} G_{p}\left(X_{p}\right)\right\}
$$

where the expectation is taken with respect to the Markov chain with initial distribution $X_{0} \sim M_{0}$ and transitions $X_{p} \sim M_{p}\left(X_{p-1}, \cdot\right)$, establishes the connection to Feynman-Kac formulae. Measures with the structure in (1)-(2) arise in a variety of statistical contexts.

\subsection{Motivating examples of Feynman-Kac models}

As a first example, consider a hidden Markov model: a bivariate Markov chain $\left(X_{p}, Y_{p}\right)_{p=0, \ldots, n}$ where $\left(X_{p}\right)_{p=0, \ldots, n}$ is itself Markov with initial distribution $M_{0}$ and transitions $X_{p} \sim M_{p}\left(X_{p-1}, \cdot\right)$, and such that each $Y_{p}$ is conditionally independent of $\left(X_{q}, Y_{q} ; q \neq p\right)$ given $X_{p}$. If the conditional distribution of $Y_{p}$ given $X_{p}$ admits a density $g_{p}\left(X_{p}, \cdot\right)$ and one fixes a sequence of observed values $y_{0}, \ldots, y_{n-1}$, then with $G_{p}\left(x_{p}\right)=g_{p}\left(x_{p}, y_{p}\right), \eta_{n}$ is the conditional distribution of $X_{n}$ given $y_{0}, \ldots, y_{n-1}$. Hence, $\eta_{n}(\varphi)$ is a conditional expectation and $\gamma_{n}(\mathbf{X})=\gamma_{n}(1)$ is the marginal likelihood of $y_{0}, \ldots, y_{n-1}$

As a second example, consider the following sequential simulation setup. Let $\pi_{0}$ and $\pi_{1}$ be two probability measures on $(\mathbf{X}, \mathcal{X})$ such that $\pi_{0}(d x)=\bar{\pi}_{0}(x) d x / Z_{0}$ and $\pi_{1}(d x)=\bar{\pi}_{1}(x) d x / Z_{1}$, where $\bar{\pi}_{0}$ and $\bar{\pi}_{1}$ are unnormalized probability densities with respect to a common dominating measure $d x$ and $Z_{i}=\int_{\mathrm{X}} \bar{\pi}_{i}(x) d x, i \in\{0,1\}$ are integrals unavailable in closed form. In Bayesian statistics $\pi_{1}$ may arise as a posterior distribution from which one wishes to sample, e.g. having multiple modes 
and complicated local covariance structures, $\pi_{0}$ is a more benign distribution from which sampling is feasible, and calculating $Z_{1} / Z_{0}$ allows assessment of model fit. Introducing a sequence $0=\beta_{0}<\cdots<$ $\beta_{n}=1$ and taking $G_{p}(x)=\left\{\bar{\pi}_{1}(x) / \bar{\pi}_{0}(x)\right\}^{\beta_{p+1}-\beta_{p}}, M_{0}=\pi_{0}$ and for each $p=1, \ldots, n, M_{p}$ as a Markov kernel invariant with respect to the distribution with density proportional to $\bar{\pi}_{0}(x)^{1-\beta_{p}} \bar{\pi}_{1}(x)^{\beta_{p}}$, one obtains by elementary manipulations

$$
\gamma_{p}(S)=\frac{1}{Z_{0}} \int_{S} \bar{\pi}_{0}(x)^{1-\beta_{p}} \bar{\pi}_{1}(x)^{\beta_{p}} d x, \quad \eta_{n}=\pi_{1}, \quad \gamma_{n}(\mathrm{X})=\frac{Z_{1}}{Z_{0}}
$$

so that $\eta_{1}, \ldots, \eta_{n-1}$ forms a sequence of intermediate distributions between $\pi_{0}$ and $\pi_{1}$. This type of construction appears in [Del Moral et al., 2006] and references therein.

\subsection{Particle approximations}

We now introduce particle approximations of the measures in (1)-(2). Let $c_{0: n}$ be a sequence of positive real numbers and $N \in \mathbb{N}$. We define a sequence of particle numbers $N_{0: n}$ by $N_{p}=\left\lceil c_{p} N\right\rceil$ for $p \in\{0, \ldots, n\}$. To avoid notational complications, we shall assume throughout that $c_{0: n}$ and $N$ are such that $\min _{p} N_{p} \geq 2$. The particle system consists of a sequence $\zeta=\zeta_{0: n}$, where for each $p$, $\zeta_{p}=\left(\zeta_{p}^{1}, \ldots, \zeta_{p}^{N_{p}}\right)$ and each $\zeta_{p}^{i}$ is valued in $\mathrm{X}$. To describe the resampling operation we also introduce random variables denoting the indices of the ancestors of each random variable $\zeta_{p}^{i}$. That is, for each $i \in\left[N_{p}\right], A_{p-1}^{i}$ is a $\left[N_{p-1}\right]$-valued random variable and we write $A_{p-1}=\left(A_{p-1}^{1}, \ldots, A_{p-1}^{N_{p}}\right)$ for $p \in[n]$ and $A=A_{0: n-1}$.

A simple algorithmic description of the particle system is given in Algorithm 1. An important and non-standard feature here is that we keep track of a collection of Eve indices $E_{0: n}$ with $E_{p}=$ $\left(E_{p}^{1}, \ldots, E_{p}^{N_{p}}\right)$ for each $p$, which will be put to use in our variance estimators. We adopt the Eve terminology because $E_{p}^{i}$ represents the index of the time 0 ancestor of $\zeta_{p}^{i}$. The fact that $N_{p}$ may vary with $p$ is also atypical, and allows us to address asymptotically optimal particle allocation in Section 6.1. On a first reading, one may wish to assume that $N_{0: n}$ is not time-varying, i.e. $c_{p}=1$ so $N_{p}=N$ for all $p \in\{0, \ldots, n\}$. Figure 1 is a graphical representation of a realization of a small particle system.

Algorithm 1. The particle filter.

1. At time 0: for each $i \in\left[N_{0}\right]$, sample $\zeta_{0}^{i} \sim M_{0}(\cdot)$ and set $E_{0}^{i} \leftarrow i$.

2. At each time $p=1, \ldots, n$ : for each $i \in\left[N_{p}\right]$,

(a) Sample $A_{p-1}^{i} \sim \mathcal{C}\left\{G_{p-1}\left(\zeta_{p-1}^{1}\right), \ldots, G_{p-1}\left(\zeta_{p-1}^{N_{p-1}}\right)\right\}$.

(b) Sample $\zeta_{p}^{i} \sim M_{p}\left(\zeta_{p-1}^{A_{p-1}^{i}}, \cdot\right)$ and set $E_{p}^{i} \leftarrow E_{p-1}^{A_{p-1}^{i}}$.

The particle approximations of $\eta_{n}$ and $\gamma_{n}$ are defined respectively, with the convention $\prod_{p=0}^{-1} \eta_{p}^{N}\left(G_{p}\right)=$ 1 , by the random measures

$$
\eta_{n}^{N}=\frac{1}{N_{n}} \sum_{i \in\left[N_{n}\right]} \delta_{\zeta_{n}^{i}}, \quad \gamma_{n}^{N}=\left\{\prod_{p=0}^{n-1} \eta_{p}^{N}\left(G_{p}\right)\right\} \eta_{n}^{N}
$$

and we observe that, similar to $(2), \eta_{n}^{N}=\gamma_{n}^{N} / \gamma_{n}^{N}(1)$. To simplify presentation, the dependence of $\gamma_{n}^{N}$ and $\eta_{n}^{N}$ on $c_{0: n}$ is suppressed from the notation. The following proposition establishes basic properties of the particle approximations, which validate their use.

Proposition 1. There exists a map $\sigma_{n}^{2}: \mathcal{L}(\mathcal{X}) \rightarrow[0, \infty)$ such that for any $\varphi \in \mathcal{L}(\mathcal{X}):$ 


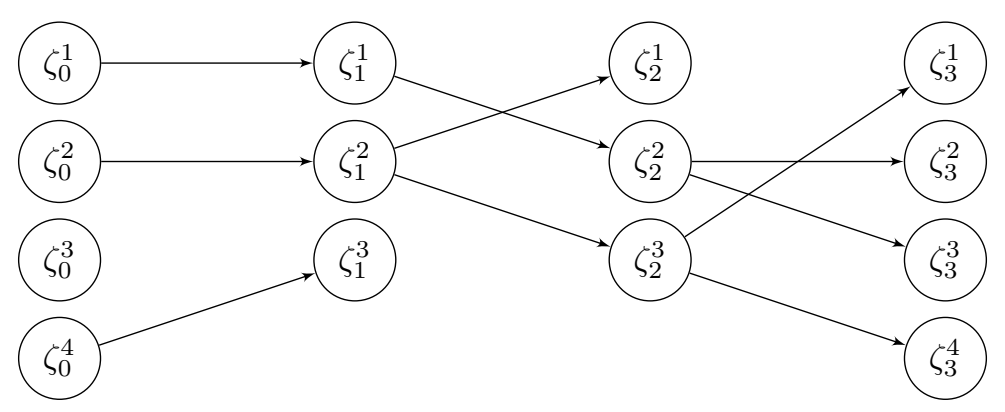

Figure 1: A particle system with $n=3$ and $N_{0: 3}=(4,3,3,4)$. An arrow from $\zeta_{p-1}^{i}$ to $\zeta_{p}^{j}$ indicates that the ancestor of $\zeta_{p}^{j}$ is $\zeta_{p-1}^{i}$, i.e. $A_{p-1}^{j}=i$. In the realization shown, the ancestral indices are $A_{0}=$ $(1,2,4), A_{1}=(2,1,2)$ and $A_{2}=(3,2,2,3)$, while the Eve indices are $E_{0}=(1,2,3,4), E_{1}=(1,2,4)$, $E_{2}=(2,1,2)$ and $E_{3}=(2,1,1,2)$.

1. $E\left\{\gamma_{n}^{N}(\varphi)\right\}=\gamma_{n}(\varphi)$, for all $N \geq 1$,

2. $\gamma_{n}^{N}(\varphi) \rightarrow \gamma_{n}(\varphi)$ almost surely and $N \operatorname{var}\left\{\gamma_{n}^{N}(\varphi) / \gamma_{n}(1)\right\} \rightarrow \sigma_{n}^{2}(\varphi)$,

3. $\eta_{n}^{N}(\varphi) \rightarrow \eta_{n}(\varphi)$ almost surely and $N E\left[\left\{\eta_{n}^{N}(\varphi)-\eta_{n}(\varphi)\right\}^{2}\right] \rightarrow \sigma_{n}^{2}\left(\varphi-\eta_{n}(\varphi)\right)$.

In the case that the number of particles is constant over time, $N_{p}=N$, these properties are well known and can be deduced, for example, from various results of Del Moral [2004]. The arguments used to treat the general $N_{p}=\left\lceil c_{p} N\right\rceil$ case are not substantially different, but since they seem not to have been published anywhere in exactly the form we need, we include a proof of Proposition 1 in the supplement.

\subsection{A variance estimator}

For $\varphi \in \mathcal{L}(\mathcal{X})$, consider the quantity

$$
V_{n}^{N}(\varphi)=\eta_{n}^{N}(\varphi)^{2}-\left(\prod_{p=0}^{n-1} \frac{N_{p}}{N_{p}-1}\right) \frac{1}{N_{n}\left(N_{n}-1\right)} \sum_{i, j: E_{n}^{i} \neq E_{n}^{j}} \varphi\left(\zeta_{n}^{i}\right) \varphi\left(\zeta_{n}^{j}\right),
$$

which is readily computable as a byproduct of Algorithm 1. The following theorem is the first main result of the paper. We state it here to make some of the practical implications of our work accessible to the reader before entering into more technical details; it shows that via (4), the Eve variables $E_{n}^{i}$ can be used to estimate the Monte Carlo errors associated with $\gamma_{n}^{N}(\varphi)$ and $\eta_{n}^{N}(\varphi)$.

Theorem 1. The following hold for any $\varphi \in \mathcal{L}(\mathcal{X})$, with $\sigma_{n}^{2}(\cdot)$ as in Proposition 1:

1. $E\left\{\gamma_{n}^{N}(1)^{2} V_{n}^{N}(\varphi)\right\}=\operatorname{var}\left\{\gamma_{n}^{N}(\varphi)\right\}$ for all $N \geq 1$,

2. $N V_{n}^{N}(\varphi) \rightarrow \sigma_{n}^{2}(\varphi)$ in probability,

3. $N V_{n}^{N}\left(\varphi-\eta_{n}^{N}(\varphi)\right) \rightarrow \sigma_{n}^{2}\left(\varphi-\eta_{n}(\varphi)\right)$ in probability.

The proof of Theorem 1, given in the appendix, relies on a number of intermediate results concerning moment properties of the particle approximations which we shall develop in the coming sections. Before embarking on this development let us discuss how (4) may be interpreted. Consider random variables $X^{1}, \ldots, X^{N}$ with sample mean $\bar{X}$ and sample variance

$$
\bar{X}^{2}-\frac{1}{N(N-1)} \sum_{i \neq j} X^{i} X^{j}=\frac{1}{N(N-1)} \sum_{i}\left(X^{i}-\bar{X}\right)^{2} .
$$


When $X^{1}, \ldots, X^{N}$ are independent and identically distributed it is of course elementary that (5) is an unbiased estimator of $\operatorname{var}(\bar{X})$ and consistency properties are easily established. Observe the resemblance between (4) and the left hand side of (5). Some of the features which distinguish these two expressions, notably the summation over $\left\{(i, j): E_{n}^{i} \neq E_{n}^{j}\right\}$ and the product term in (4), are a reflection of the dependence between the particles and specific distributional characteristics of Algorithm 1. One of the main difficulties we face is to develop a suitable mathematical perspective from which to describe this dependence and thus establish that (4) does indeed have the properties stated in Theorem 1.

It seems natural to ask if (4) can be re-written so as to resemble the right hand side of (5) and thus be interpreted as some kind of sample variance across the population of particles. This motivates the following corollary, using the notation

$$
\#_{n}^{i}=\operatorname{card}\left\{j: E_{n}^{j}=i\right\}, \quad \Delta_{n}^{i}=\frac{1}{\#_{n}^{i}} \sum_{j: E_{n}^{j}=i} \varphi\left(\zeta_{n}^{j}\right)-\eta_{n}^{N}(\varphi),
$$

with the convention $\Delta_{n}^{i}=0$ when $\#_{n}^{i}=0$. Recall from Section 2.3 that in the hidden Markov model and sequential simulation examples $\gamma_{n}(1)$ is respectively the marginal likelihood and ratio of normalizing constants, hence our interest in $V_{n}^{N}(\varphi)$ with specifically $\varphi=1$.

Corollary 1. In the case that $c_{p}=1$ for all $p \in\{0, \ldots, n\}$,

$$
\begin{aligned}
& N V_{n}^{N}(1)=\frac{1}{N} \sum_{i \in[N]}\left(\#_{n}^{i}-1\right)^{2}-n+\mathcal{O}_{p}(1 / N), \\
& N V_{n}^{N}\left(\varphi-\eta_{n}^{N}(\varphi)\right)=\frac{1}{N} \sum_{i \in[N]}\left(\#_{n}^{i} \Delta_{n}^{i}\right)^{2}+\mathcal{O}_{p}(1 / N) .
\end{aligned}
$$

The proof is in the supplement.

Since $\sum_{i} \#_{n}^{i}=N$, the first term on the right hand side of (6) can be interpreted as a sample variance of the $\#_{n}^{i}$ 's, reflecting variation in the numbers of time $n$ descendants across the population of time 0 particles. Since $\sum_{i} \#_{n}^{i} \Delta_{n}^{i}=0$, the first term on the right hand side of (7) can be interpreted as a sample variance which reflects both variation in the $\#_{n}^{i}$ 's and the deviations of the familial means $\left(\#_{n}^{i}\right)^{-1} \sum_{j: E_{n}^{j}=i} \varphi\left(\zeta_{n}^{j}\right)$ from the population mean $\eta_{n}^{N}(\varphi)$. Note that when $n=0, E_{0}^{i}=i$ always and

$$
V_{0}^{N}\left(\varphi-\eta_{0}^{N}(\varphi)\right)=\frac{1}{N_{0}\left(N_{0}-1\right)} \sum_{i \in\left[N_{0}\right]}\left\{\varphi\left(\zeta_{0}^{i}\right)-\eta_{0}^{N}(\varphi)\right\}^{2},
$$

which is in keeping with $\zeta_{0}^{i}$ being independent and identically distributed according to $\eta_{0}$.

\section{Moment properties of the particle approximations}

\subsection{Genealogical tracing variables}

Our next step is to introduce some auxiliary random variables associated with the genealogical structure of the particle system. These auxiliary variables are introduced only for purposes of analysis: they will assist in deriving and justifying our variance estimators. Given $(A, \zeta)$, the first collection of variables, $K^{1}=\left(K_{0}^{1}, \ldots, K_{n}^{1}\right)$, is conditionally distributed as follows: $K_{n}^{1}$ is uniformly distributed on $\left[N_{n}\right]$ and for each $p=n-1, \ldots, 0, K_{p}^{1}=A_{p}^{K_{p+1}^{1}}$. Given $(A, \zeta)$ and $K^{1}$, the second collection of variables, $K^{2}=\left(K_{0}^{2}, \ldots, K_{n}^{2}\right)$, is conditionally distributed as follows: $K_{n}^{2}$ is uniformly distributed on $\left[N_{n}\right]$ and for each $p=n-1, \ldots, 0$ we have $K_{p}^{2}=A_{p}^{K_{p+1}^{2}}$ if $K_{p+1}^{2} \neq K_{p+1}^{1}$ and $K_{p}^{2} \sim \mathcal{C}\left(G_{p}\left(\zeta_{p}^{1}\right), \ldots, G_{p}\left(\zeta_{p}^{N_{p}}\right)\right)$ if $K_{p+1}^{2}=K_{p+1}^{1}$. The interpretation of $K^{1}$ is that it traces backwards in time the ancestral lineage of a particle chosen randomly from the population at time $n . K^{2}$ is slightly more complicated: it traces backwards in time a sequence of broken ancestral lineages, where breaks in the lineages occur when components of $K^{1}$ and $K^{2}$ coincide. 


\subsection{Lack-of-bias and second moment of $\gamma_{n}^{N}(\varphi)$}

We now give expressions for the first two moments of $\gamma_{n}^{N}(\varphi)$.

Lemma 1. For any $\varphi \in \mathcal{L}(\mathcal{X}), E\left\{\gamma_{n}^{N}(1) \varphi\left(\zeta_{n}^{K_{n}^{1}}\right)\right\}=\gamma_{n}(\varphi)$ and $E\left\{\gamma_{n}^{N}(\varphi)\right\}=\gamma_{n}(\varphi)$.

The proof is in the supplement. This lack-of-bias property $E\left\{\gamma_{n}^{N}(\varphi)\right\}=\gamma_{n}(\varphi)$ is quite well known and a martingale proof for the $N_{p}=N$ case can be found, for example, in Del Moral [2004, Ch. 9].

In order to present an expression for the second moment of $\gamma_{n}^{N}(\varphi)$, we now introduce a collection of measures on $\mathcal{X}^{\otimes 2}$, denoted $\left\{\mu_{b}: b \in B_{n}\right\}$ where $B_{n}=\{0,1\}^{n+1}$ is the set of binary strings of length $n+1$. The measures are constructed as follows. For a given $b \in B_{n}$, let $\left(X_{p}, X_{p}^{\prime}\right)_{0 \leq p \leq n}$ be a Markov chain with state-space $X^{2}$, distributed according to the following recipe. If $b_{0}=0$ then $X_{0} \sim M_{0}$ and $X_{0}^{\prime} \sim M_{0}$ independently, while if $b_{0}=1$ then $X_{0}^{\prime}=X_{0} \sim M_{0}$. Then, for $p=1, \ldots, n$, if $b_{p}=0$ then $X_{p} \sim M_{p}\left(X_{p-1}, \cdot\right)$ and $X_{p}^{\prime} \sim M_{p}\left(X_{p-1}^{\prime}, \cdot\right)$ independently, while if $b_{p}=1$ then $X_{p}^{\prime}=X_{p} \sim M_{p}\left(X_{p-1}, \cdot\right)$. Letting $E_{b}$ denote expectation with respect to the law of this Markov chain we then define

$$
\mu_{b}(S)=E_{b}\left[\mathbb{I}\left\{\left(X_{n}, X_{n}^{\prime}\right) \in S\right\} \prod_{p=0}^{n-1} G_{p}\left(X_{p}\right) G_{p}\left(X_{p}^{\prime}\right)\right], \quad S \in \mathcal{X}^{\otimes 2}, \quad b \in B_{n} .
$$

Similarly to $(3)$ we shall write $\mu_{b}(\varphi)=E_{b}\left\{\varphi\left(X_{n}, X_{n}^{\prime}\right) \prod_{p=0}^{n-1} G_{p}\left(X_{p}\right) G_{p}\left(X_{p}^{\prime}\right)\right\}$, for $\varphi \in \mathcal{L}\left(\mathcal{X}^{\otimes 2}\right)$ and $b \in B_{n}$.

Remark 1. Observe that with $0_{n} \in B_{n}$ denoting the zero string, $\mu_{0_{n}}\left(\varphi^{\otimes 2}\right)=\gamma_{n}(\varphi)^{2}$.

Let $\left[N_{0: n}\right]=\left[N_{0}\right] \times \cdots \times\left[N_{n}\right]$, and for any $b \in B_{n}$,

$$
\mathcal{I}(b)=\left\{\left(k^{1}, k^{2}\right) \in\left[N_{0: n}\right]^{2}: \text { for each } p, k_{p}^{1}=k_{p}^{2} \Longleftrightarrow b_{p}=1\right\}
$$

which is the set of pairs of $\left[N_{0: n}\right]$-valued strings which coincide in their $p$-th coordinate exactly when $b_{p}=1$.

Lemma 2. For any $\varphi \in \mathcal{L}\left(\mathcal{X}^{\otimes 2}\right)$ and $b \in B_{n}$,

$$
E\left[\mathbb{I}\left\{\left(K^{1}, K^{2}\right) \in \mathcal{I}(b)\right\} \gamma_{n}^{N}(1)^{2} \varphi\left(\zeta_{n}^{K_{n}^{1}}, \zeta_{n}^{K_{n}^{2}}\right)\right]=\prod_{p=0}^{n}\left\{\left(\frac{1}{N_{p}}\right)^{b_{p}}\left(1-\frac{1}{N_{p}}\right)^{1-b_{p}}\right\} \mu_{b}(\varphi)
$$

and

$$
E\left\{\gamma_{n}^{N}(\varphi)^{2}\right\}=\sum_{b \in B_{n}} \prod_{p=0}^{n}\left(\frac{1}{N_{p}}\right)^{b_{p}}\left(1-\frac{1}{N_{p}}\right)^{1-b_{p}} \mu_{b}\left(\varphi^{\otimes 2}\right)
$$

The proof of Lemma 2 is in the supplement and uses an argument involving the law of a doubly conditional sequential Monte Carlo algorithm [see also Andrieu et al., 2016]. The identity (9) was first proved by Cérou et al. [2011] in the case where $N_{p}=N$. Our proof technique is different: we obtain (9) as a consequence of (8). The appearance of $K^{1}, K^{2}$ in (8) is also central to the justification of our variance estimators below.

\subsection{Asymptotic variances}

For each $p \in\{0, \ldots, n\}$, we denote by $e_{p} \in B_{n}$ the vector with a 1 in position $p$ and zeros elsewhere. As in Remark $1,0_{n}$ denotes the zero string in $B_{n}$. The following result builds upon Lemmas 1-2. It shows that a particular subset of the measures $\left\{\mu_{b}: b \in B_{n}\right\}$, namely $\mu_{0_{n}}$ and $\left\{\mu_{e_{p}}: p=0, \ldots, n\right\}$, appear in the asymptotic variances. 
Lemma 3. For any $\varphi \in \mathcal{L}(\mathcal{X})$, define

$$
v_{p, n}(\varphi)=\frac{\mu_{e_{p}}\left(\varphi^{\otimes 2}\right)-\mu_{0_{n}}\left(\varphi^{\otimes 2}\right)}{\gamma_{n}(1)^{2}}, \quad p \in\{0, \ldots, n\} .
$$

Then $N \operatorname{var}\left\{\gamma_{n}^{N}(\varphi) / \gamma_{n}(1)\right\} \rightarrow \sum_{p=0}^{n} c_{p}^{-1} v_{p, n}(\varphi)$ and

$$
N E\left[\left\{\eta_{n}^{N}(\varphi)-\eta_{n}(\varphi)\right\}^{2}\right] \rightarrow \sum_{p=0}^{n} c_{p}^{-1} v_{p, n}\left(\varphi-\eta_{n}(\varphi)\right) .
$$

The proof of Lemma 3 is in the supplement.

Remark 2. In light of Lemma 3, the map $\sigma_{n}^{2}$ in Proposition 1 satisfies

$$
\sigma_{n}^{2}(\varphi)=\sum_{p=0}^{n} c_{p}^{-1} v_{p, n}(\varphi), \quad \varphi \in \mathcal{L}(\mathcal{X})
$$

An expression for $v_{p, n}(\varphi)$ in terms of $\left(M_{p}, G_{p}\right)_{0 \leq p \leq n}$ is obtained by observing that if we define

$$
Q_{p}\left(x_{p-1}, \mathrm{~d} x_{p}\right)=G_{p-1}\left(x_{p-1}\right) M_{p}\left(x_{p-1}, \mathrm{~d} x_{p}\right), \quad p \in\{1, \ldots, n\},
$$

and $Q_{n, n}=I d, Q_{p, n}=Q_{p+1} \cdots Q_{n}$ for $p \in\{0, \ldots, n-1\}$, then $\mu_{e_{p}}(\varphi)=\gamma_{p}\left(Q_{p, n}(\varphi)^{2}\right)$. In combination with Remark 1, we obtain

$$
v_{p, n}(\varphi)=\frac{\gamma_{p}(1) \gamma_{p}\left(Q_{p, n}(\varphi)^{2}\right)}{\gamma_{n}(1)^{2}}-\eta_{n}(\varphi)^{2}=\frac{\eta_{p}\left(Q_{p, n}(\varphi)^{2}\right)}{\eta_{p} Q_{p, n}(1)^{2}}-\eta_{n}(\varphi)^{2} .
$$

\section{The estimators}

\subsection{Particle approximations of each $\mu_{b}$}

We now introduce particle approximations of the measures $\left\{\mu_{b}: b \in B_{n}\right\}$, from which we shall subsequently derive the variance estimators. For each $b \in B_{n}$, and $\varphi \in \mathcal{L}\left(\mathcal{X}^{\otimes 2}\right)$ we define

$$
\mu_{b}^{N}(\varphi)=\left[\prod_{p=0}^{n}\left(N_{p}\right)^{b_{p}}\left(\frac{N_{p}}{N_{p}-1}\right)^{1-b_{p}}\right] \gamma_{n}^{N}(1)^{2} E\left[\mathbb{I}\left\{\left(K^{1}, K^{2}\right) \in \mathcal{I}(b)\right\} \varphi\left(\zeta_{n}^{K_{n}^{1}}, \zeta_{n}^{K_{n}^{2}}\right) \mid A, \zeta\right] .
$$

Recalling from Section 3.1 that given $A$ and $\zeta, K_{n}^{1}$ and $K_{n}^{2}$ are conditionally independent and each uniformly distributed on $\left[N_{n}\right]$, it follows from (14) that

$$
\begin{aligned}
\gamma_{n}^{N}(\varphi)^{2} & =\gamma_{n}^{N}(1)^{2} \frac{1}{N_{n}^{2}} \sum_{i, j \in\left[N_{n}\right]} \varphi\left(\zeta_{n}^{i}\right) \varphi\left(\zeta_{n}^{j}\right) \\
& =\gamma_{n}^{N}(1)^{2} \sum_{b \in B_{n}} E\left[\mathbb{I}\left\{\left(K^{1}, K^{2}\right) \in \mathcal{I}(b)\right\} \varphi\left(\zeta_{n}^{K_{n}^{1}}\right) \varphi\left(\zeta_{n}^{K_{n}^{2}}\right) \mid A, \zeta\right] \\
& =\sum_{b \in B_{n}}\left\{\prod_{p=0}^{n}\left(\frac{1}{N_{p}}\right)^{b_{p}}\left(1-\frac{1}{N_{p}}\right)^{1-b_{p}}\right\} \mu_{b}^{N}\left(\varphi^{\otimes 2}\right),
\end{aligned}
$$

mirroring (9). This identity is complemented by the following result.

Theorem 2. For any $b \in B_{n}$ and $\varphi \in \mathcal{L}\left(\mathcal{X}^{\otimes 2}\right)$,

1. $E\left\{\mu_{b}^{N}(\varphi)\right\}=\mu_{b}(\varphi)$ for all $N \geq 1$, 
2. $\sup _{N \geq 1} N E\left[\left\{\mu_{b}^{N}(\varphi)-\mu_{b}(\varphi)\right\}^{2}\right]<\infty$ and hence $\mu_{b}^{N}(\varphi) \rightarrow \mu_{b}(\varphi)$ in probability.

The proof of Theorem 2 is in the supplement. Although (14) can be computed in principle from the output of Algorithm 1 without the need for any further simulation, the conditional expectation in (14) involves a summation over all binary strings in $\mathcal{I}(b)$, so calculating $\mu_{b}^{N}\left(\varphi^{\otimes 2}\right)$ in practice may be computationally expensive. Fortunately, relatively simple and computationally efficient expressions are available for $\mu_{b}^{N}\left(\varphi^{\otimes 2}\right)$ in the cases $b=0_{n}$ and $b=e_{p}$, and those are the only ones required to construct our variance estimators.

\subsection{Variance estimators}

Our next objective is to explain how (4) is related to the measures $\mu_{b}^{N}$ and to introduce another family of estimators associated with the individual terms in (12). We need the following technical lemma.

Lemma 4. The following identity of events holds: $\left\{E_{n}^{K_{n}^{1}} \neq E_{n}^{K_{n}^{2}}\right\}=\left\{\left(K^{1}, K^{2}\right) \in \mathcal{I}\left(0_{n}\right)\right\}$.

The proof is in the appendix. Combined with the fact that given $(A, \zeta), K_{n}^{1}, K_{n}^{2}$ are independent and identically distributed according to the uniform distribution on $\left[N_{n}\right]$, we have

$$
E\left[\mathbb{I}\left\{\left(K^{1}, K^{2}\right) \in \mathcal{I}\left(0_{n}\right)\right\} \varphi\left(\zeta_{n}^{K_{n}^{1}}, \zeta_{n}^{K_{n}^{2}}\right) \mid A, \zeta\right]=N_{n}^{-2} \sum_{i, j: E_{n}^{i} \neq E_{n}^{j}} \varphi\left(\zeta_{n}^{i}\right) \varphi\left(\zeta_{n}^{j}\right)
$$

and therefore we arrive at the following equivalent of (4), written in terms of $\mu_{0_{n}}^{N}$,

$$
V_{n}^{N}(\varphi)=\eta_{n}^{N}(\varphi)^{2}-\frac{\mu_{0_{n}}^{N}\left(\varphi^{\otimes 2}\right)}{\gamma_{n}^{N}(1)^{2}}
$$

Detailed pseudocode for computing $V_{n}^{N}(\varphi)$ in $\mathcal{O}(N)$ time and space upon running Algorithm 1 is provided in the supplement.

Mirroring (10), we now define

$$
v_{p, n}^{N}(\varphi)=\frac{\mu_{e_{p}}^{N}\left(\varphi^{\otimes 2}\right)-\mu_{0_{n}}^{N}\left(\varphi^{\otimes 2}\right)}{\gamma_{n}^{N}(1)^{2}}, \quad p \in\{0, \ldots, n\}, \quad v_{n}^{N}(\varphi)=\sum_{p=0}^{n} c_{p}^{-1} v_{p, n}^{N}(\varphi) .
$$

Detailed pseudocode for computing each $v_{p, n}^{N}(\varphi)$ and $v_{n}^{N}(\varphi)$ with time and space complexity in $\mathcal{O}(N n)$ time upon running Algorithm 1 is provided in the supplement. The time complexity is the same as that of running Algorithm 1, but the space complexity is larger. Empirically, we have found that $N V_{n}^{N}(\varphi)$ is very similar to $v_{n}^{N}(\varphi)$ as an estimator of $\sigma_{n}^{2}(\varphi)$ when $N$ is large enough that they are both accurate, and hence may be preferable due to its reduced space complexity.

Theorem 3. For any $\varphi \in \mathcal{L}(\mathcal{X})$,

1. $E\left\{\gamma_{n}^{N}(1)^{2} v_{p, n}^{N}(\varphi)\right\}=\gamma_{n}(1)^{2} v_{p, n}(\varphi)$ for all $N \geq 1$,

2. $v_{p, n}^{N}(\varphi) \rightarrow v_{p, n}(\varphi)$ and $v_{p, n}^{N}\left(\varphi-\eta_{n}^{N}(\varphi)\right) \rightarrow v_{p, n}\left(\varphi-\eta_{n}(\varphi)\right)$, both in probability,

3. $E\left\{\gamma_{n}^{N}(1)^{2} v_{n}^{N}(\varphi)\right\}=\gamma_{n}(1)^{2} \sigma_{n}^{2}(\varphi)$ for all $N \geq 1$ and $v_{n}^{N}(\varphi) \rightarrow \sigma_{n}^{2}(\varphi)$ in probability.

\section{Estimators for updated measures}

In some applications there is interest in approximating the updated measures:

$$
\hat{\gamma}_{n}(S)=\int_{S} G_{n}(x) \gamma_{n}(\mathrm{~d} x), \quad \hat{\eta}_{n}(S)=\frac{\hat{\gamma}_{n}(S)}{\hat{\gamma}_{n}(1)}, \quad S \in \mathcal{X} .
$$


In the hidden Markov model setting described in Section 2.2, e.g., $\hat{\eta}_{n}$ is the conditional distribution of $X_{n}$ given $y_{0}, \ldots, y_{n}$, that is $\hat{\eta}_{n}$ is a filtering distribution, while $\eta_{n}$ is a predictive distribution.

The updated particle approximations are defined by

$$
\hat{\gamma}_{n}^{N}(S)=\int_{S} G_{n}(x) \gamma_{n}^{N}(\mathrm{~d} x), \quad \hat{\eta}_{n}^{N}(S)=\frac{\hat{\gamma}_{n}^{N}(S)}{\hat{\gamma}_{n}^{N}(1)}, \quad S \in \mathcal{X},
$$

and we now define their variance estimators. To facilitate this task, we consider a fixed $\varphi \in \mathcal{L}(\mathcal{X})$, and define $\hat{\varphi}(x)=G_{n}(x) \varphi(x)$. The following relationships can then be deduced: $\hat{\gamma}_{n}(\varphi) \equiv \gamma_{n}(\hat{\varphi})$, $\hat{\eta}_{n}(\varphi) \equiv \eta_{n}(\hat{\varphi}) / \eta_{n}\left(G_{n}\right), \hat{\gamma}_{n}^{N}(\varphi) \equiv \gamma_{n}^{N}(\hat{\varphi})$ and $\hat{\eta}_{n}^{N}(\varphi) \equiv \eta_{n}^{N}(\hat{\varphi}) / \eta_{n}^{N}\left(G_{n}\right)$. We define analogues of $\sigma_{n}^{2}$ and $v_{p, n}$ for the updated particle approximations as

$$
\hat{\sigma}_{n}^{2}(\varphi)=\lim _{N \rightarrow \infty} N \operatorname{var}\left\{\hat{\gamma}_{n}^{N}(\varphi) / \hat{\gamma}_{n}(1)\right\}, \quad \hat{v}_{p, n}(\varphi)=\frac{v_{p, n}(\hat{\varphi})}{\eta_{n}\left(G_{n}\right)^{2}}
$$

and the proposition below is a counterpart to Proposition 1 and Lemma 3.

Proposition 2. For any $\varphi \in \mathcal{L}(\mathcal{X})$,

1. $\hat{\gamma}_{n}^{N}(\varphi) \rightarrow \hat{\gamma}_{n}(\varphi)$ almost surely and $\hat{\sigma}_{n}^{2}(\varphi)=\sum_{p=0}^{n} c_{p}^{-1} \hat{v}_{p, n}(\varphi)$,

2. $\hat{\eta}_{n}^{N}(\varphi) \rightarrow \hat{\eta}_{n}(\varphi)$ almost surely and $N E\left[\left\{\hat{\eta}_{n}^{N}(\varphi)-\hat{\eta}_{n}(\varphi)\right\}^{2}\right] \rightarrow \hat{\sigma}_{n}^{2}\left(\varphi-\hat{\eta}_{n}(\varphi)\right)$.

The proofs of Proposition 2, and Theorems 4-5 below can be found in the supplement. Proposition 2 implies the relationship $\hat{\sigma}_{n}^{2}(\varphi)=\sigma_{n}^{2}(\hat{\varphi}) / \eta_{n}\left(G_{n}\right)^{2}$. The corresponding estimates of the variance, asymptotic variance and the terms therein are now obtained and analogues of Theorems 1 and 3 follow straightforwardly. Below we write the estimators $\hat{V}_{n}^{N}, \hat{v}_{p, n}^{N}$ etc. in terms of $V_{n}^{N}, \eta_{n}^{N}$ and $v_{p, n}^{N}$ to emphasize that the same algorithms can be used to compute them, just as $\hat{\gamma}_{n}^{N}(\varphi)$ and $\hat{\eta}_{n}^{N}(\varphi)$ can be computed as $\gamma_{n}^{N}(\hat{\varphi})$ and $\eta_{n}^{N}(\hat{\varphi}) / \eta_{n}^{N}\left(G_{n}\right)$, respectively.

Theorem 4. For any $\varphi \in \mathcal{L}(\mathcal{X})$, with

$$
\hat{V}_{n}^{N}(\varphi)=V_{n}^{N}(\hat{\varphi}) / \eta_{n}^{N}\left(G_{n}\right)^{2},
$$

1. $E\left\{\hat{\gamma}_{n}^{N}(1)^{2} \hat{V}_{n}^{N}(\varphi)\right\}=\operatorname{var}\left\{\hat{\gamma}_{n}^{N}(\varphi)\right\}$ for all $N \geq 1$,

2. $N \hat{V}_{n}^{N}(\varphi) \rightarrow \hat{\sigma}_{n}^{2}(\varphi)$ in probability,

3. $N \hat{V}_{n}^{N}\left(\varphi-\hat{\eta}_{n}^{N}(\varphi)\right) \rightarrow \hat{\sigma}_{n}^{2}\left(\varphi-\hat{\eta}_{n}(\varphi)\right)$ in probability.

Remark 3. It follows from (4), (17), (18) and simple manipulations that

$$
\frac{N \hat{V}_{n}^{N}\left(\varphi-\hat{\eta}_{n}^{N}(\varphi)\right)}{\left(\prod_{p=0}^{n} \frac{N_{p}}{N_{p}-1}\right)}=N \sum_{i \in\left[N_{0}\right]}\left[\frac{\sum_{j \in\left[N_{n}\right]: E_{n}^{j}=i} G_{n}\left(\zeta_{n}^{j}\right)\left\{\varphi\left(\zeta_{n}^{j}\right)-\hat{\eta}_{n}^{N}(\varphi)\right\}}{\sum_{j \in\left[N_{n}\right]} G_{n}\left(\zeta_{n}^{j}\right)}\right]^{2},
$$

the right hand side of which is, in the case where $N$ is not time-varying, precisely the estimator in Equation 2.9 of Chan and Lai [2013].

Theorem 5. For any $\varphi \in \mathcal{L}(\mathcal{X})$, with

$$
\hat{v}_{p, n}^{N}(\varphi)=v_{p, n}^{N}(\hat{\varphi}) / \eta_{n}^{N}\left(G_{n}\right)^{2}, \quad \hat{v}_{n}^{N}(\varphi)=\sum_{p=0}^{n} c_{p}^{-1} \hat{v}_{p, n}^{N}(\varphi),
$$

1. $E\left\{\hat{\gamma}_{n}^{N}(1)^{2} \hat{v}_{p, n}^{N}(\varphi)\right\}=\hat{\gamma}_{n}(1)^{2} \hat{v}_{p, n}(\varphi)$ for all $N \geq 1$,

2. $\hat{v}_{p, n}^{N}(\varphi) \rightarrow \hat{v}_{p, n}(\varphi)$ and $\hat{v}_{p, n}^{N}\left(\varphi-\hat{\eta}_{n}^{N}(\varphi)\right) \rightarrow \hat{v}_{p, n}\left(\varphi-\hat{\eta}_{n}(\varphi)\right)$, both in probability,

3. $E\left\{\hat{\gamma}_{n}^{N}(1)^{2} \hat{v}_{n}^{N}(\varphi)\right\}=\hat{\gamma}_{n}(1)^{2} \hat{\sigma}_{n}^{2}(\varphi)$ for all $N \geq 1$ and $\hat{v}_{n}^{N}(\varphi) \rightarrow \hat{\sigma}_{n}^{2}(\varphi)$ in probability. 


\section{Use of the estimators to tune the particle filter}

The variance estimators we have proposed can of course be applied directly to report estimates of Monte Carlo error alongside particle approximations. Estimates of quantities such as $v_{p, n}(\varphi)$ may also aid algorithm and design. We provide here two simple examples of adaptive methods to illustrate this, firstly concerning how to improve performance by allowing particle numbers to vary over time, and secondly concerning how to choose particle numbers so as to achieve some user-defined performance criterion. To simplify presentation, we focus on performance in estimating $\gamma_{n}^{N}(\varphi)$, the ideas can easily be modified easily to deal instead with $\eta_{n}^{N}(\varphi), \hat{\gamma}_{n}^{N}(\varphi)$ or $\hat{\eta}_{n}^{N}(\varphi)$.

\subsection{Asymptotically optimal allocation}

The following well known result is closely related to Neyman's optimal allocation in stratified random sampling [Tschuprow, 1923, Neyman, 1934]. A short proof using Jensen's inequality can be found in Glasserman [2004, Section 4.3].

Lemma 5. Let $a_{0}, \ldots, a_{n} \geq 0$. The function $\left(c_{0}, \ldots, c_{n}\right) \mapsto \sum_{p=0}^{n} c_{p}^{-1} a_{p}$ is minimized, subject to the constraints $\min _{p} c_{p}>0$ and $\sum_{p=0}^{n} c_{p}=n+1$, at $(n+1)^{-1}\|a\|_{2}^{2}$ when $c_{p} \propto a_{p}^{1 / 2}$.

As a consequence, we can in principle minimize $\sigma_{n}^{2}(\varphi)$ by choosing $c_{p} \propto v_{p, n}(\varphi)^{1 / 2}$. An approximation of this optimal allocation can be obtained by the following two-stage procedure. First run a particle filter with $N_{p}=N$ to obtain the estimates $v_{p, n}^{N}(\varphi)$ and then define $c_{0: n}$ by $c_{p}=\max \left\{v_{p, n}^{N}(\varphi), g(N)\right\}$, where $g$ is some positive but decreasing function with $\lim _{N \rightarrow \infty} g(N)=0$. Then run a second particle filter with each $N_{p}=\left\lceil c_{p} N\right\rceil$, and report the quantities of interest, e.g., $\gamma_{n}^{N}(\varphi)$. The function $g$ is chosen to ensure that $c_{p}>0$ and that for large $N$ we permit small values of $c_{p}$. The quantity $\sum_{p=0}^{n} v_{p, n}^{N}(\varphi) /\left(\sum_{p=0}^{n} c_{p}^{-1} v_{p, n}^{N}(\varphi)\right)$, obtained from the first run, is an indication of the improvement in variance obtained by using the new allocation.

Approximately optimal allocation has previously been addressed by Bhadra and Ionides [2016], who introduced a meta-model to approximate the distribution of the Monte Carlo error associated with $\log \gamma_{n}^{N}(1)$ in terms of an autoregressive process, the objective function to be minimized then being the variance under this meta-model. They provide only empirical evidence for the fit of their meta-model, whereas our approach targets the true asymptotic variance $\sigma_{n}^{2}(\varphi)$ directly.

\subsection{An adaptive particle filter}

Monte Carlo errors of particle filter approximations can be sensitive to $N$, and an adequate value of $N$ to achieve a given error may not be known a priori. The following procedure increases $N$ until $V_{n}^{N}(\varphi)$ is in a given interval.

Consider the case where we wish to estimate $\gamma_{n}(\varphi)$. Given an initial number of particles $N^{(0)}$ and a threshold $\delta>0$, one can run successive particle filters, doubling the number of particles each time, until the associated random variable $V_{n}^{N^{(\tau)}}(\varphi) \in[0, \delta]$. Finally, one runs a final particle filter with $N^{(\tau)}$ particles, and returns the estimate of interest. We provide empirical evidence in Section 7 that this procedure can be effective in some applications.

\section{Applications and illustrations}

In this section we demonstrate the empirical performance of the estimators we have proposed in three examples. Our numerical results mostly address the accuracy of our estimators of the asymptotic variance $\sigma_{n}^{2}(\varphi)$, the individual terms $v_{p, n}(\varphi)$, and the effectiveness of the applications described in Section 6 with the test functions $\varphi \equiv 1$ and $\varphi=I d$, the identity function. Where the results for later examples are qualitatively similar to those of the first, the corresponding figures can be found in the supplement. 


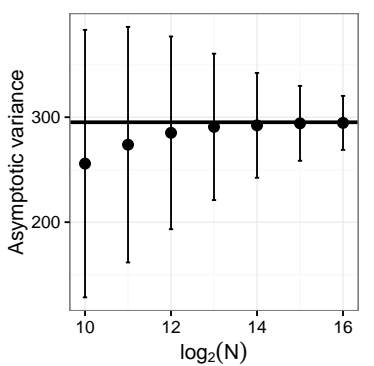

(a) $\varphi \equiv 1$

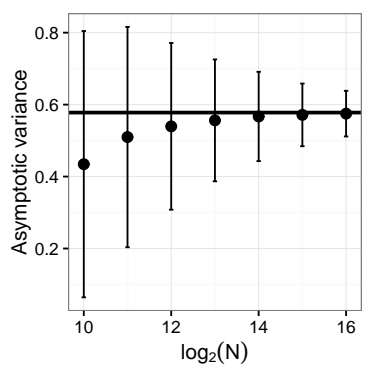

(b) $\varphi=I d-\hat{\eta}_{n}^{N}(I d)$

Figure 2: Estimated asymptotic variances $N \hat{V}_{n}^{N}(\varphi)$ (dots and error bars for the mean \pm one standard deviation from $10^{4}$ replicates) against $\log _{2} N$ for the linear Gaussian example. The horizontal lines correspond to the true asymptotic variances. The sample variances of $\hat{\gamma}_{n}^{N}(1) / \hat{\gamma}_{n}(1)$ and $\hat{\eta}_{n}^{N}(I d)$, scaled by $N$, were close to their asymptotic variances.

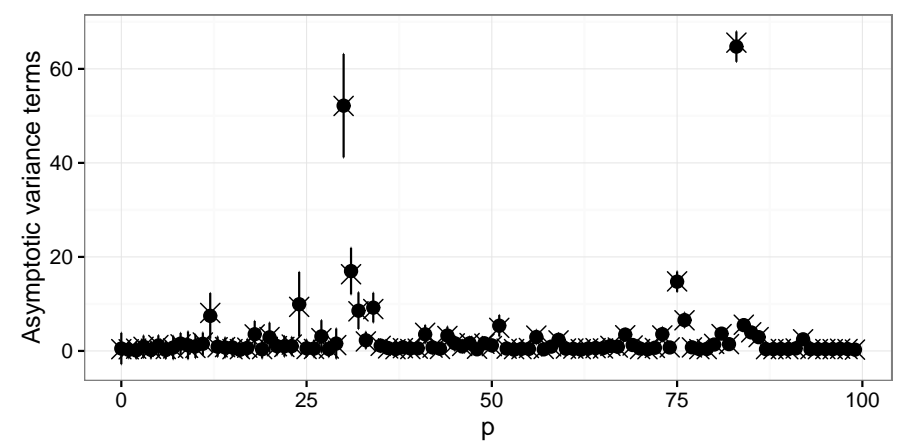

Figure 3: Plot of $\hat{v}_{p, n}^{N}(1)$ (dots and error bars for the mean \pm one standard deviation from $10^{4}$ replicates) and $\hat{v}_{p, n}(1)$ (crosses) at each $p \in\{0, \ldots, n\}$ for the Linear Gaussian example, with $N=10^{5}$.

\subsection{Linear Gaussian hidden Markov model}

This model is specified by $M_{0}(\cdot)=\mathcal{N}(\cdot ; 0,1), M_{p}\left(x_{p-1}, \cdot\right)=\mathcal{N}\left(\cdot ; 0.9 x_{p-1}, 1\right)$ and $G_{p}\left(x_{p}\right)=\mathcal{N}\left(y_{p} ; x_{p}, 1\right)$. The measures $\hat{\eta}_{n}$ and $\hat{\gamma}_{n}$ are available in closed form via the Kalman filter, and for suitable $\varphi$ the quantities $\hat{v}_{p, n}(\varphi)$ etc. can be computed exactly, allowing us to assess the accuracy of our estimators. We used a synthetic dataset, simulated according to the model with $n=99$. A Monte Carlo study with $10^{4}$ replicates of $\hat{V}_{n}^{N}(\varphi)$ for each value of $N$ and $c_{p} \equiv 1$ was used to measure the accuracy of the estimate $N \hat{V}_{n}^{N}(\varphi)$ as $N$ grows; results are displayed in Figure 2 and for this data $\hat{\sigma}_{n}^{2}(1)=295.206$ and $\hat{\sigma}_{n}^{2}\left(I d-\hat{\eta}_{n}(I d)\right) \approx 0.58$. The estimates $\hat{v}_{n}^{N}(\varphi)$ differed very little from $N \hat{V}_{n}^{N}(\varphi)$, and so are not shown. We then tested the accuracy of the estimates $\hat{v}_{p, n}^{N}(1)$; results are displayed in Figure 3 . The estimates $\hat{v}_{p, n}^{N}\left(I d-\hat{\eta}_{n}^{N}(I d)\right)$ are very close to 0 for $p<95$ and with values $(0.0017,0.012,0.082,0.48)$ for $p \in\{96,97,98,99\}$; this behaviour is in keeping with time-uniform bounds on asymptotic variances obtained by Whiteley [2013], see also references therein.

We also compared a constant $N$ particle filter, the asymptotically optimal particle filter where the asymptotically optimal allocation is computed exactly, and its approximation described in Section 6.1 for different values of $N$ using a Monte Carlo study with $10^{4}$ replicates. We took $g(N)=2 / \log _{2} N$ in defining the approximation, and the results in Figure 4a indicate that indeed the approximation reduces the variance. The improvement is fairly modest for this particular model, and indeed the exact asymptotic variances associated with the constant $N$ and asymptotically optimal particle filters differ by less than a factor of 2 . In contrast, Figure $4 \mathrm{~b}$ shows that the improvement can be fairly dramatic 


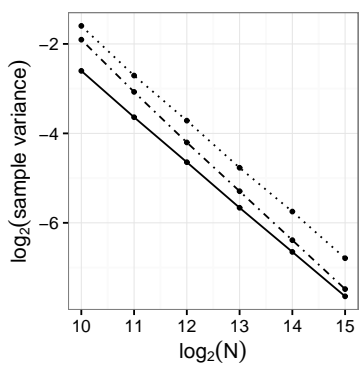

(a)

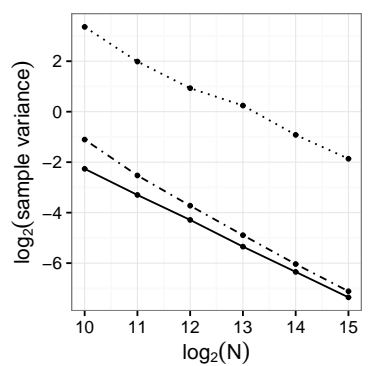

(b)

Figure 4: Logarithmic plots of the sample variance across $10^{4}$ replicates of $\gamma_{n}^{N}(1) / \gamma_{n}(1)$ against $N$ for the linear Gaussian example, using a constant $N$ particle filter (dotted), the approximation to the asymptotically optimal particle filter (dot-dash), and the asymptotically optimal particle filter (solid). In Figure $4 \mathrm{~b}$, the observation sequence is $y_{p}=0$ for $p \in\{0, \ldots, 99\} \backslash\{49\}$ and $y_{49}=8$.

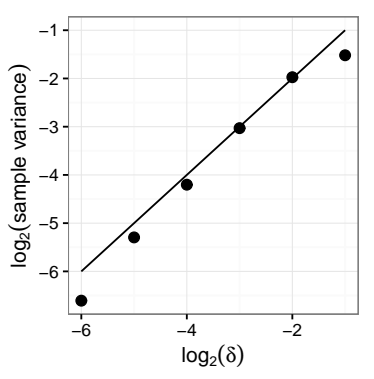

(a)

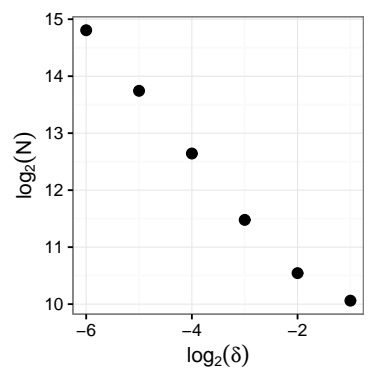

(b)

Figure 5: Logarithmic plots for the simple adaptive $N$ particle filter estimates of $\hat{\gamma}_{n}(1)$ for the linear Gaussian example. Figure (a) plots the sample variance of $\hat{\gamma}_{n}^{N}(1) / \hat{\gamma}_{n}(1)$ against $\delta$, with the straight line $y=x$. Figure (b) plots $N$ against $\delta$, where $N$ is the average number of particles used by the final particle filter.

in the presence of outlying observations; the improvement in variance there is by a factor of around 40. Finally, we tested the adaptive particle filter described in Section 6.2 using $10^{3}$ replicates for each value of $\delta$; results are displayed in Figure 5, and indicate that the estimates of $\hat{\gamma}_{n}(1)$ are close to their prescribed thresholds.

\subsection{Stochastic volatility hidden Markov model}

A stochastic volatility model is defined by $M_{0}(\cdot)=\mathcal{N}\left\{\cdot ; 0, \sigma^{2} /\left(1-\rho^{2}\right)\right\}, M_{p}\left(x_{p-1}, \cdot\right)=\mathcal{N}\left(\cdot ; \rho x_{p-1}, \sigma^{2}\right)$ and $G_{p}\left(x_{p}\right)=\mathcal{N}\left(y_{p} ; 0, \beta^{2} \exp \left(x_{p}\right)\right)$. We used the pound/dollar daily exchange rates for 100 consecutive weekdays ending on 28th June, 1985, a subset of the well-known dataset analyzed in Harvey, Ruiz and Shephard (1994). Our results are obtained by choosing the parameters $(\rho, \sigma, \beta)=(0.95,0.25,0.5)$. We provide in the supplement plots of the accuracy of the estimate $N \hat{V}_{n}^{N}(\varphi)$ as $N$ grows using $10^{4}$ replicates for each value of $N$; the asymptotic variances $\hat{\sigma}_{n}^{2}(1)$ and $\hat{\sigma}_{n}^{2}\left(I d-\hat{\eta}_{n}(I d)\right)$ are estimated as being approximately 354 and 1.31 respectively. In the supplement we plot the estimates of $\hat{v}_{p, n}(\varphi)$. We found modest improvement for the approximation of the asymptotically optimal particle filter, as one could infer from the estimated $\hat{v}_{p, n}(\varphi)$. For the simple adaptive $N$ particle filter, results are provided in the supplement, and indicate that the estimates of $\hat{\gamma}_{n}(1)$ are close to their prescribed thresholds. 


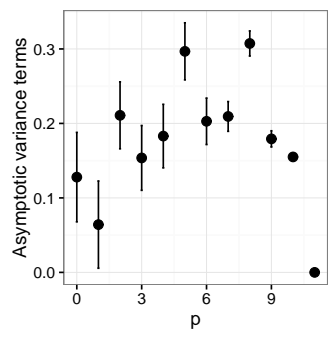

(a) $\varphi \equiv 1, k=10$

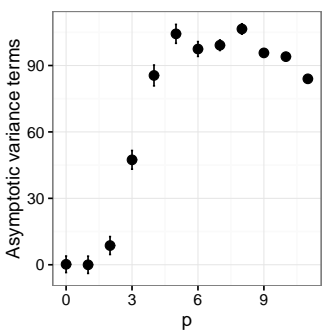

(b) $\varphi=I d-\eta_{n}(I d), k=$

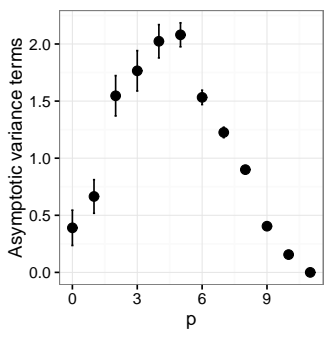

(c) $\varphi \equiv 1, k=1$

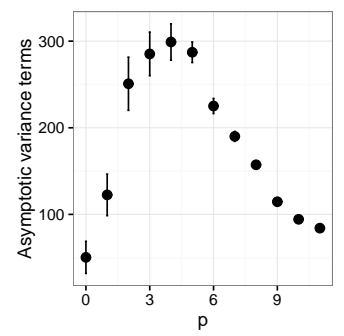

(d) $\varphi=I d-\eta_{n}(I d), k=1$

Figure 6: Plot of $v_{p, n}^{N}(\varphi)$ (dots and error bars for the mean \pm one standard deviation) at each $p \in$ $\{0, \ldots, n\}$ with $k=10$ iterations (a)-(b) and $k=1$ iteration (c)-(d) for each Markov kernel in the SMC sampler example and $N=10^{5}$.

\subsection{An SMC sampler}

We consider a sequential simulation problem, as described in Section 2.3, with $\mathrm{X}=\mathbb{R}, \bar{\pi}_{0}(x)=$ $\mathcal{N}\left(0,10^{2}\right)$ and $\bar{\pi}_{1}(x)=0.3 \mathcal{N}\left(x ;-10,0.1^{2}\right)+0.7 \mathcal{N}\left(x ; 10,0.2^{2}\right)$. The distribution $\pi_{1}$ is bi-modal with well-separated modes. With $n=11$, and the sequence of tempering parameters

$$
\beta_{0: n}=(0,0.0005,0.001,0.0025,0.005,0.01,0.025,0.05,0.1,0.25,0.5,1),
$$

we let each Markov kernel $M_{p}, p \in\{1, \ldots, n\}$ be an $\eta_{p}$-invariant random walk Metropolis kernel iterated $k=10$ times with proposal variance $\tau_{p}^{2}$, where $\tau_{1: n}=(10,9,8,7,6,5,4,3,2,1,1)$.

One striking difference between the estimates for this model and those for the hidden Markov models above is that the asymptotic variance $\sigma_{n}^{2}\left(I d-\eta_{n}(I d)\right) \approx 822$ is considerably larger than $\sigma_{n}^{2}(1) \approx 2.1$; the variability of the estimates $N V_{n}^{N}(\varphi)$ is shown in the supplement. Inspection of the estimates of $v_{p, n}(\varphi)$ in Figures 6 allows us to investigate both this difference and the dependence of $v_{p, n}(\varphi)$ on $k$ in greater detail.

In Figure 6(a)-(b) we can see that while $v_{p, n}(1)$ is small for all $p$, the values of $v_{p, n}\left(I d-\eta_{n}(I d)\right)$ are larger for large $p$ than for small $p$; this could be due to the inability of the Metropolis kernels $\left(M_{q}\right)_{q \geq p}$ to mix well due to the separation of the modes in $\left(\eta_{q}\right)_{q \geq p}$ when $p$ is large. In Figure 6(c)-(d), $k=1$, that is each $M_{P}$ consists of only a single iterate of a Metropolis kernel, and we see that the values of $v_{p, n}(\varphi)$ associated with small $p$ are much larger than when $k=10$, indicating that the larger number of iterates does improve the asymptotic variance of the particle approximations. However, the impact on $v_{p, n}(\varphi)$ is less pronounced for large $p$. Results for the simple adaptive $N$ particle filter approximating $\eta_{n}(I d)$ are provided in the supplement, which again show that the estimates are close to their prescribed thresholds.

\section{Discussion}

\subsection{Alternatives to the bootstrap particle filter}

In the hidden Markov model examples above, we have constructed the Feynman-Kac measures taking $M_{0}, \ldots, M_{n}$ to be the initial distribution and transition probabilities of the latent process and defining $G_{0}, \ldots, G_{n}$ to incorporate the realized observations. This is only one, albeit important, way to construct particle approximations of $\eta_{n}$, and the algorithm itself is usually referred to as the bootstrap particle filter. Alternative specifications of $\left(M_{p}, G_{p}\right)_{0 \leq p \leq n}$ lead to different Feynman-Kac models, as discussed in Del Moral [2004, Section 2.4.2], and the variance estimators introduced here are applicable to these models as well. 


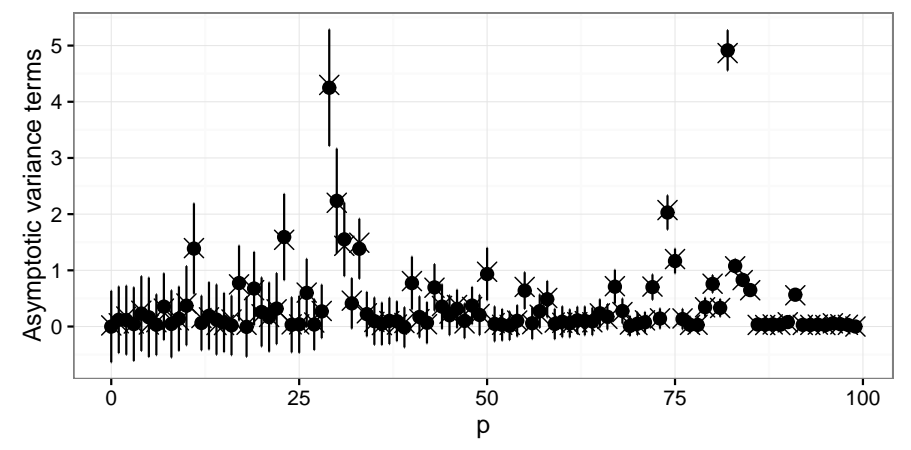

Figure 7: Plot of $\check{v}_{p, n}^{N}(1)$ (dots and error bars for the mean \pm one standard deviation) and $\check{v}_{p, n}(1)$ (crosses) at each $p \in\{0, \ldots, n\}$ in the Linear Gaussian example.

One particular specification corresponds to the "fully adapted" auxiliary particle filter of Pitt and Shephard [1999], as discussed by Doucet and Johansen [2008]. Specifically, we define $\check{M}_{0}\left(\mathrm{~d} x_{0}\right)=M_{0}\left(\mathrm{~d} x_{0}\right) G_{0}\left(x_{0}\right) / M_{0}\left(G_{0}\right)$, and

$$
\check{M}_{p}\left(x_{p-1}, \mathrm{~d} x_{p}\right)=\frac{M_{p}\left(x_{p-1}, \mathrm{~d} x_{p}\right) G_{p}\left(x_{p}\right)}{M_{p}\left(G_{p}\right)\left(x_{p-1}\right)}, \quad p \in[n],
$$

and then $\check{G}_{0}\left(x_{0}\right)=M_{0}\left(G_{0}\right) M_{1}\left(G_{1}\right)\left(x_{0}\right)$ and $\check{G}_{p}\left(x_{p}\right)=M_{p+1}\left(G_{p+1}\right)\left(x_{p}\right), p \geq 1$. If we denote by $\check{\gamma}_{n}$ and $\check{\eta}_{n}$ the Feynman-Kac measures associated with $\left(\check{M}_{p}, \breve{G}_{p}\right)_{0 \leq p \leq n}$, we obtain $\check{\gamma}_{n}=\hat{\gamma}_{n}$ and $\check{\eta}_{n}=\hat{\eta}_{n}$. Moreover, the variances of $\check{\gamma}_{n}^{N}(\varphi)$ and $\check{\eta}_{n}^{N}(\varphi)$ are often smaller than the variances of $\hat{\gamma}_{n}^{N}(\varphi)$ and $\hat{\eta}_{n}^{N}(\varphi)$. In Figure 7, we plot the corresponding $\breve{v}_{p, n}(1)$ and their approximations for the same linear Gaussian example in Section 7.1. Here, the asymptotic variance of $\check{\gamma}_{n}^{N}(1) / \check{\gamma}_{n}(1)$ is 40.718 , more than 7 times smaller than $\hat{\sigma}_{n}^{2}(1)$.

\subsection{Estimators based on i.i.d. replicates}

It is clearly possible to estimate consistently the variance of $\gamma_{n}^{N}(\varphi) / \gamma_{n}(1)$ by using i.i.d. replicates of $\gamma_{n}^{N}$. Such estimates necessarily entail simulation of multiple particle filters. We now compare the accuracy of such estimates with those based on i.i.d. replicates of $V_{n}^{N}(\varphi)$. For some $\varphi \in \mathcal{L}(\mathcal{X})$ and $B \in \mathbb{N}$, let $\gamma_{n, i}^{N}(\varphi)$ and $V_{n, i}^{N}(\varphi)$ be i.i.d. replicates for $i \in[B]$, and define $M=N^{-1} \sum_{i \in[B]} \gamma_{n, i}^{N}(1)$. A standard estimate of $\operatorname{var}\left\{\gamma_{n}^{N}(\varphi) / \gamma_{n}(1)\right\}$ is obtained by calculating the sample variance of $\left\{M^{-1} \gamma_{n, i}^{N}(\varphi) ; i \in[B]\right\}$. Noting the lack-of-bias of $\gamma_{n}^{N}(1)^{2} V_{n}^{N}(\varphi)$, an alternative estimate of $\operatorname{var}\left[\gamma_{n}^{N}(\varphi) / \gamma_{n}(1)\right]$ can be obtained as $\frac{1}{B} \sum_{i \in[B]}\left[M^{-1} \gamma_{n, i}^{N}(1)\right]^{2} V_{n, i}^{N}(\varphi)$. Both these estimates can be seen as ratios of simple Monte Carlo estimates of $\operatorname{var}\left\{\gamma_{n}^{N}(\varphi)\right\}$ and $\gamma_{n}(1)^{2}$, and are therefore consistent as $B \rightarrow \infty$. We show in Figure 8 a comparison between these estimates for the three models discussed in Section 7 with $N=10^{3}$ and $\varphi \equiv 1$, and we can see that the alternative estimate based on $\hat{V}_{n}^{N}(1)$ is empirically more accurate for these examples.

\subsection{Final remarks}

The particular approximations developed here provide a natural way to estimate the terms appearing in the non-asymptotic second moment expression (9). To the best of our knowledge, we have also provided the first generally applicable, consistent estimators of $v_{p, n}(\varphi)$. The expression (9) does not apply to particle approximations with resampling schemes other than multinomial, and one possible avenue of future research is to investigate estimators in these other settings. Whilst we have emphasized variances and asymptotic variances, the measures $\mu_{b}$ also appear in expressions which describe propagation of chaos properties of the particle system. For instance, in the situation $N_{p} \equiv N$, the asymptotic bias 

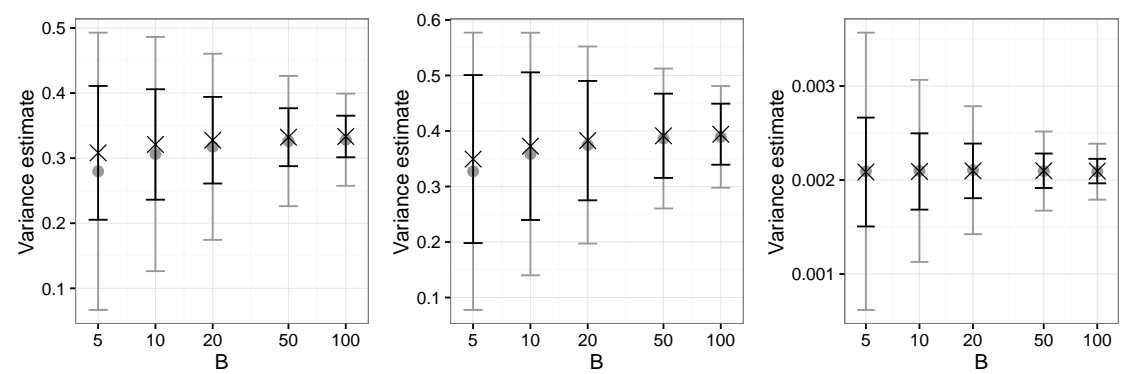

Figure 8: Plot of the standard estimate of $\operatorname{var}\left[\hat{\gamma}_{n}^{N}(\varphi) / \hat{\gamma}_{n}(1)\right]$ (gray dots and error bars) and the alternative estimate using $\hat{V}_{n}^{N}(1)$ (black crosses and error bars) against $B$ in (left to right) the examples of Sections 7.1-7.3.

formula of Del Moral et al. [2007, p.7.] can be expressed as

$$
N E\left\{\eta_{n}^{N}(\varphi)-\eta_{n}(\varphi)\right\} \rightarrow-\sum_{p=0}^{n-1} \frac{\eta_{p}\left\{Q_{p, n}(1) Q_{p, n}\left(\varphi-\eta_{n}(\varphi)\right)\right\}}{\eta_{p} Q_{p, n}(1)^{2}} \equiv-\sum_{p=0}^{n-1} \frac{\mu_{e_{p}}\left\{1 \otimes\left(\varphi-\eta_{n}(\varphi)\right)\right\}}{\gamma_{n}(1)^{2}}
$$

which could be consistently estimated by replacing $\mu_{e_{p}}$ and $\gamma_{n}(1)$ by $\mu_{e_{p}}^{N}$ and $\gamma_{n}^{N}(1)$. Finally, the technique used in the proof of Lemma 2 can be generalized to obtain expressions for arbitrary positive integer moments of $\gamma_{n}^{N}(\varphi)$.

\section{Supplementary Material}

The supplementary material at http://www.warwick.ac.uk/alee/vestpf_supp.pdf includes algorithms for efficient computation of the variance estimators, and proofs of Corollary 1, Lemmas 1-3, Propositions 1-2, and Theorems 2, 4 and 5.

\section{Appendix}

Proof of Theorem 1. Throughout the proof, $\rightarrow$ denotes convergence in probability. For part 1., the fact $\mu_{0_{n}}\left(\varphi^{\otimes 2}\right)=\gamma_{n}(\varphi)^{2}$ and Theorem 2 together give

$$
E\left\{\gamma_{n}^{N}(1)^{2} V_{n}^{N}(\varphi)\right\}=E\left\{\gamma_{n}^{N}(\varphi)^{2}-\mu_{0_{n}}^{N}\left(\varphi^{\otimes 2}\right)\right\}=E\left\{\gamma_{n}^{N}(\varphi)^{2}\right\}-\gamma_{n}(\varphi)^{2}=\operatorname{var}\left\{\gamma_{n}^{N}(\varphi)\right\}
$$

For part 2., combining the identity (15), $\mu_{b}^{N}\left(\varphi^{\otimes 2}\right) \rightarrow \mu_{b}\left(\varphi^{\otimes 2}\right)$ by Theorem 2, and the fact that for any $b \in B_{n}$ other than $0_{n}$ and $e_{0}, \ldots, e_{n}, \prod_{p=0}^{n}\left(\frac{1}{N_{p}}\right)^{b_{p}}\left(1-\frac{1}{N_{p}}\right)^{1-b_{p}}$ is in $\mathcal{O}\left(N^{-2}\right)$, we obtain

$$
\gamma_{n}^{N}(\varphi)^{2}-\mu_{0_{n}}^{N}\left(\varphi^{\otimes 2}\right)=\left\{\sum_{p=0}^{n} \frac{\mu_{e_{p}}^{N}\left(\varphi^{\otimes 2}\right)-\mu_{0_{n}}^{N}\left(\varphi^{\otimes 2}\right)}{\left\lceil c_{p} N\right\rceil}\right\}+\mathcal{O}_{p}\left(N^{-2}\right) .
$$

Also noting that by Proposition $1 \gamma_{n}^{N}(1)^{2} \rightarrow \gamma_{n}(1)^{2}$, from (10) that $\gamma_{n}(1)^{2} v_{p, n}(\varphi)=\mu_{e_{p}}\left(\varphi^{\otimes 2}\right)-$ $\mu_{0_{n}}\left(\varphi^{\otimes 2}\right)$ and again using $\mu_{b}^{N}\left(\varphi^{\otimes 2}\right) \rightarrow \mu_{b}\left(\varphi^{\otimes 2}\right)$, we then have

$$
N V_{n}^{N}(\varphi)=\frac{N}{\gamma_{n}^{N}(1)^{2}}\left\{\gamma_{n}^{N}(\varphi)^{2}-\mu_{0_{n}}^{N}\left(\varphi^{\otimes 2}\right)\right\} \rightarrow \sum_{p=0}^{n} \frac{v_{p, n}(\varphi)}{c_{p}}=\sigma_{n}^{2}(\varphi) .
$$


For part 3., first note that by Theorem 2 and Proposition 1 , for any $b \in B_{n}$,

$$
\begin{aligned}
\mu_{b}^{N}\left(\left[\varphi-\eta_{n}^{N}(\varphi)\right]^{\otimes 2}\right) & =\mu_{b}^{N}\left(\varphi^{\otimes 2}\right)-\eta_{n}^{N}(\varphi)\left[\mu_{b}^{N}(\varphi \otimes 1)+\mu_{b}^{N}(1 \otimes \varphi)\right]+\eta_{n}^{N}(\varphi)^{2} \mu_{b}^{N}\left(1^{\otimes 2}\right) \\
& \rightarrow \mu_{b}\left(\left[\varphi-\eta_{n}(\varphi)\right]^{\otimes 2}\right)
\end{aligned}
$$

from which it follows that (19) also holds with $\varphi$ replaced by $\varphi-\eta_{n}^{N}(\varphi)$, and then

$$
N V_{n}^{N}\left(\varphi-\eta_{n}^{N}(\varphi)\right) \rightarrow \sum_{p=0}^{n} \frac{v_{p, n}\left(\varphi-\eta_{n}(\varphi)\right)}{c_{p}}=\sigma_{n}^{2}\left(\varphi-\eta_{n}(\varphi)\right)
$$

similarly to $(20)$.

Proof of Lemma 4. For $i \in\left[N_{n}\right]$ define $B_{n-1}^{i}=A_{n-1}^{i}$ and $B_{p-1}^{i}=A_{p-1}^{B_{p}^{i}}$ for $p \in[n-1]$. Since in Algorithm $1, E_{p}^{i}=E_{p-1}^{A_{p-1}^{i}}$ for all $p \in[n], i \in\left[N_{p}\right]$, a simple inductive argument then shows that

$$
E_{n}^{i}=E_{p}^{B_{p}^{i}}, \quad p \in\{0, \ldots, n\}, i \in\left[N_{n}\right] .
$$

We shall now prove $\left(K^{1}, K^{2}\right) \in \mathcal{I}\left(0_{n}\right) \Rightarrow E_{n}^{K_{n}^{1}} \neq E_{n}^{K_{n}^{2}}$. Recall from Section 3.1 that when $\left(K^{1}, K^{2}\right) \in$ $\mathcal{I}\left(0_{n}\right)$, we have $A_{p-1}^{K_{p}^{1}}=K_{p-1}^{1} \neq K_{p-1}^{2}=A_{p-1}^{K_{p}^{2}}$ for all $p \in[n]$, hence $B_{0}^{K_{n}^{1}}=K_{0}^{1} \neq K_{0}^{2}=B_{0}^{K_{n}^{2}}$. Applying (21) with $p=0$ and using the fact that in Algorithm $1, E_{0}^{i}=i$ for all $i \in\left[N_{n}\right]$, we have $E_{n}^{i}=E_{0}^{B_{0}^{i}}=B_{0}^{i}$, hence $E_{n}^{K_{n}^{1}}=B_{0}^{K_{n}^{1}} \neq B_{0}^{K_{n}^{2}}=E_{n}^{K_{n}^{2}}$ as required. It remains to prove $\left(K^{1}, K^{2}\right) \notin \mathcal{I}\left(0_{n}\right) \Rightarrow E_{n}^{K_{n}^{1}}=E_{n}^{K_{n}^{2}}$. Assuming $\left(K^{1}, K^{2}\right) \notin \mathcal{I}\left(0_{n}\right)$, consider $\tau=\max \left\{p: K_{p}^{1}=K_{p}^{2}\right\}$. If $\tau=n$ then clearly $E_{n}^{K_{n}^{1}}=E_{n}^{K_{n}^{2}}$, so suppose $\tau<n$. It follows from Section 3.1 that $B_{\tau}^{K_{n}^{1}}=K_{\tau}^{1}=K_{\tau}^{2}=B_{\tau}^{K_{n}^{2}}$, so taking $p=\tau$ and $i=K_{n}^{1}, K_{n}^{2}$ in (21) gives $E_{n}^{K_{n}^{1}}=E_{n}^{K_{n}^{2}}$.

Proof of Theorem 3. For part 1., Theorem 2 gives

$$
E\left\{\gamma_{n}^{N}(1)^{2} v_{p, n}^{N}(\varphi)\right\}=E\left\{\mu_{e_{p}}^{N}\left(\varphi^{\otimes 2}\right)-\mu_{0_{n}}^{N}\left(\varphi^{\otimes 2}\right)\right\}=\mu_{e_{p}}\left(\varphi^{\otimes 2}\right)-\mu_{0_{n}}\left(\varphi^{\otimes 2}\right)=\gamma_{n}(1)^{2} v_{p, n}(\varphi) .
$$

For the remainder of the proof, $\rightarrow$ denotes convergence in probability. For part $2 ., \mu_{e_{p}}^{N}\left(\varphi^{\otimes 2}\right)-$ $\mu_{0_{n}}^{N}\left(\varphi^{\otimes 2}\right) \rightarrow \gamma_{n}(1)^{2} v_{p, n}(\varphi)$ by Theorem 2 , and $\gamma_{n}^{N}(1)^{2} \rightarrow \gamma_{n}(1)^{2}$ by Proposition 1 , so $v_{p, n}^{N}(\varphi)=$ $\left[\mu_{e_{p}}^{N}\left(\varphi^{\otimes 2}\right)-\mu_{0_{n}}^{N}\left(\varphi^{\otimes 2}\right)\right] / \gamma_{n}^{N}(1)^{2} \rightarrow v_{p, n}(\varphi)$; as in the proof of Theorem 1, $\mu_{b}^{N}\left(\left[\varphi-\eta_{n}^{N}(\varphi)\right]^{\otimes 2}\right) \rightarrow$ $\mu_{b}\left(\left[\varphi-\eta_{n}(\varphi)\right]^{\otimes 2}\right)$ gives $v_{p, n}^{N}\left(\varphi-\eta_{n}^{N}(\varphi)\right) \rightarrow v_{p, n}\left(\varphi-\eta_{n}(\varphi)\right)$. Part 3. follows from parts 1 . and 2 .

\section{References}

C. Andrieu, A. Lee, and M. Vihola. Uniform ergodicity of the iterated conditional SMC and geometric ergodicity of particle Gibbs samplers. Bernoulli, 2016. , to appear.

A. Bhadra and E. L. Ionides. Adaptive particle allocation in iterated sequential Monte Carlo via approximating meta-models. Stat. Comput., 26(1):393-407, 2016.

F. Cérou, P. Del Moral, and A. Guyader. A nonasymptotic theorem for unnormalized Feynman-Kac particle models. Ann. Inst. H. Poincaré Probab. Statist., 47(3):629-649, 2011.

H. P. Chan and T. L. Lai. A general theory of particle filters in hidden Markov models and some applications. Ann. Statist., 41(6):2877-2904, 2013.

N. Chopin. Central limit theorem for sequential Monte Carlo methods and its application to Bayesian inference. Ann. Statist., 32(6):2385-2411, 2004. 
P. Del Moral. Feynman-Kac formulae: genealogical and interacting particle systems with applications. Springer Verlag, 2004.

P. Del Moral and A. Guionnet. Central limit theorem for nonlinear filtering and interacting particle systems. Ann. Appl. Probab., 9(2):275-297, 1999.

P. Del Moral and L. Miclo. Genealogies and increasing propagation of chaos for Feynman-Kac and genetic models. Ann. Appl. Probab., 11(4):1166-1198, 2001.

P. Del Moral, A. Doucet, and A. Jasra. Sequential Monte Carlo samplers. J. R. Stat. Soc. Ser. B Stat. Methodol., 68(3):411-436, 2006.

P. Del Moral, A. Doucet, and G. W. Peters. Sharp propagation of chaos estimates for Feynman-Kac particle models. Theory Probab. Appl., 51(3):459-485, 2007.

R. Douc and E. Moulines. Limit theorems for weighted samples with applications to sequential Monte Carlo methods. Ann. Statist., 36(5):2344-2376, 2008.

R. Douc, A. Guillin, and J. Najim. Moderate deviations for particle filtering. Ann. Appl. Probab., 15 (1B):587-614, 2005.

A. Doucet and A. M. Johansen. A tutorial on particle filtering and smoothing: fifteen years later. In D. Crisan and B. Rozovsky, editors, Handbook of Nonlinear Filtering. Oxford University Press, 2008.

P. Glasserman. Monte Carlo methods in financial engineering. Springer Science \& Business Media, 2004.

H. Künsch. Recursive Monte Carlo filters: algorithms and theoretical analysis. Ann. Statist., 33(5): 1983-2021, 2005.

J. Neyman. On the two different aspects of the representative method: the method of stratified sampling and the method of purposive selection. J. Roy. Statist. Soc., 97(4):558-625, 1934.

M. K. Pitt and N. Shephard. Filtering via simulation: auxiliary particle filters. J. Amer. Statist. Assoc., 94(446):590-599, 1999.

A. A. Tschuprow. On the mathematical expectation of the moments of frequency distributions in the case of correlated observations. Metron, 2:461-493, 646-683, 1923.

N. Whiteley. Stability properties of some particle filters. Ann. Appl. Prob., 23(6):2500-2537, 2013. 